\title{
Quantitative evaluation of the antiretroviral efficacy of dolutegravir
}

\author{
Sarah B. Laskey' and Robert F. Siliciano $0^{1,2}$ \\ 'Department of Medicine, Johns Hopkins University School of Medicine, Baltimore, Maryland, USA. ${ }^{2}$ Howard Hughes \\ Medical Institute, Baltimore, Maryland, USA.
}

\begin{abstract}
The second-generation HIV-1 integrase strand transfer inhibitor (InSTI) dolutegravir (DTC) has had a major impact on the treatment of HIV-1 infection. Here we describe important but previously undetermined pharmacodynamic parameters for DTC. We show that the dose-response curve slope, which indicates cooperativity and is a major determinant of antiviral activity, is higher for DTC than for first-generation InSTIs. This steepness does not reflect inhibition of multiple steps in the HIV-1 life cycle, as is the case for allosteric integrase inhibitors and HIV-1 protease inhibitors. We also show that degree of independence, a metric of interaction favorability between antiretroviral drugs, is high for DTC and nucleoside reverse transcriptase inhibitors. Finally, we demonstrate poor selective advantage for HIV-1 bearing InSTI resistance mutations. Selective advantage, which incorporates both the magnitude of resistance conferred by a mutation and its fitness cost, explains the high genetic barrier to DTC resistance. Together, these parameters provide an explanation for the remarkable clinical success of DTC.
\end{abstract}

\section{Introduction}

The second-generation HIV-1 integrase strand transfer inhibitor (InSTI) dolutegravir (DTG) has had dramatic clinical impact, demonstrating both efficacy (1-5) and a high barrier to resistance (6-8). Despite the remarkable success of DTG, its pharmacodynamic properties are incompletely understood.

The clinical efficacy of antiretroviral drugs depends on many factors. The most fundamental is intrinsic antiviral activity, which is a function of both the $\mathrm{IC}_{50}$ and the dose-response curve slope in all pharmacodynamic models $(9,10)$. Although frequently overlooked, slope is an important determinant of efficacy because of its exponential relationship with inhibition $(10,11)$. High slopes for HIV-1 protease inhibitors (12) and allosteric integrase inhibitors (ALLINIs) (13-17) reflect inhibition at multiple steps in the viral life cycle. The first-generation InSTI raltegravir has not shown activity at HIV-1 life cycle steps other than integration (13-15), but DTG has not been evaluated for such activity.

The efficacy of antiretroviral combinations also depends on the interaction between the component drugs. Two classic models for combined drug effects, Loewe additivity (18) and Bliss independence (19), predict the expected efficacies of drug combinations given the experimentally determined efficacies of individual component drugs. Loewe additivity assumes that drugs act through the same target; Bliss independence describes drugs that inhibit distinct targets. At clinical drug concentrations, the Loewe model predicts lower combined efficacy than the Bliss model. In practice, antiretroviral drugs do not interact exclusively according to either model. Degree of independence (DI), a scale defined by the Loewe and Bliss models, describes these interactions quantitatively and allows the calculation of instantaneous inhibitory potential (IIP), a metric of combined drug efficacy. IIP correlates with the clinical success of antiretroviral regimens (11), and IIP values for DTG-containing regimens have not been reported.

The genetic barrier to resistance also impacts the clinical success of antiretroviral drugs. Various point

Conflict of interest: The authors have declared that no conflict of interest exists.

Submitted: August 8, 2016 Accepted: October 18, 2016 Published: November 17, 2016

Reference information: JCI Insight. 2016;1(19):e89810. doi:10.1172/jci.insight.90033. mutations confer resistance to first-generation InSTIs, but they confer minimal resistance to DTG $(20,21)$. HIV-1 replication in the presence of DTG selects for the R263K substitution in vitro (22), but the clinical impact of this mutation is low due to its fitness cost (23). To quantify the genetic barrier to DTG resistance, we evaluated the selective advantage of InSTI resistance mutations (RMs). Selective advantage, which reflects both the degree and the fitness cost of resistance, indicates the likelihood that drug resistance will emerge in vivo.

Using an in vitro infectivity assay and quantitative analysis, we evaluated critical DTG pharmacodynamic parameters. The results of our analyses of slope, DI, IIP, and selective advantage provide a quantitative explanation for the success of DTG-containing regimens. 
A

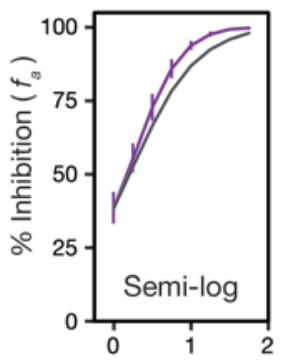

$\log ([\mathrm{DTG}] /[\mathrm{DTG}])$

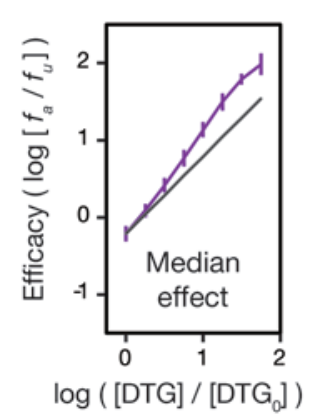

B

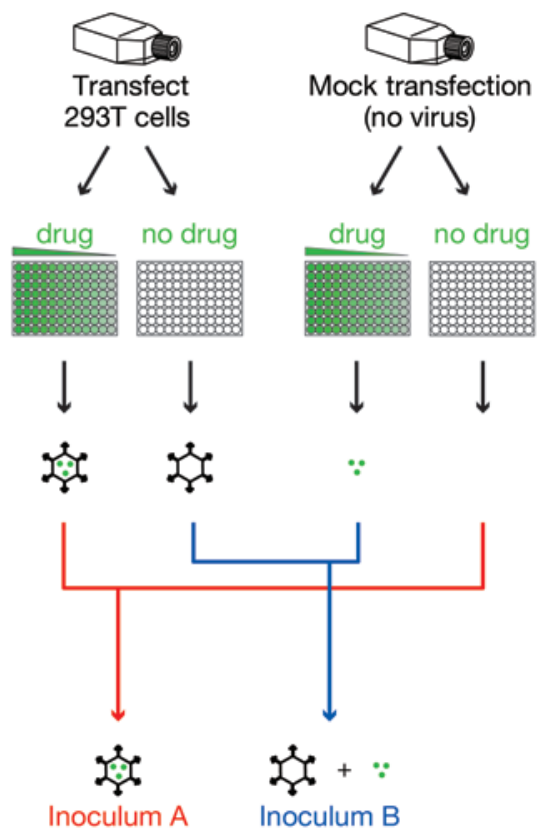

C
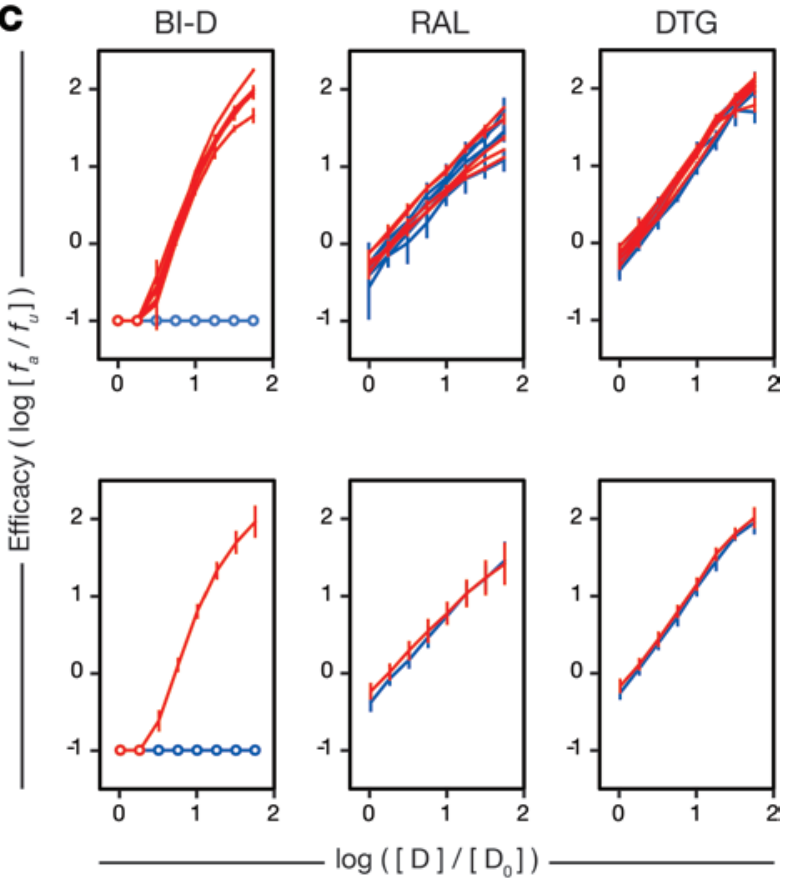

Figure 1. The steep dolutegravir (DTC) slope is not due to activity late in the HIV-1 life cycle. (A) Semi-log and median-effect curves showing the DTC dose-response relationship (purple) and a hypothetical drug with the same $\mathrm{IC}_{50}$ and a slope of $m=1$ (gray). DTC concentration is normalized to [DTG ${ }_{0}$, the empirical IC ${ }_{50}$. Logarithmic DTC concentration ( $x$ axis) is plotted against a linear (semi-log) or a logarithmic (median-effect) measure of efficacy ( $y$ axis). $f_{a}$ is the fraction of infection events affected, i.e., inhibited, by DTC at the stated concentration; $f_{u}$ is the fraction of infection events unaffected by DTC, and $f_{u}=1-f_{a}$. Error bars represent the standard deviation of 6 different blood donors. (B) Schematic of inoculum preparation for the life cycle assay, which differentiates between antiviral activity early and late in the HIV-1 life cycle. Virus is produced either in the presence or the absence of a drug, and then drug/ virus mixtures are used to infect primary CD4+ T lymphoblasts. (C) Median-effect dose-response curves for BI-D (left), raltegravir (RAL, center), and DTC (right). Open circles represent values below the detection limit. The top row shows results from 5 blood donors; error bars represent the standard deviation of 3 technical replicates. The bottom row shows results averaged over 5 blood donors in each condition; error bars represent 1 standard deviation. Reference concentrations present during virus production are: $\left[D_{0, D T C}\right]=45 \mathrm{nM} ;\left[D_{0, R A L}\right]=97 \mathrm{nM} ;\left[D_{0, B I-D}\right]=50 \mathrm{nM}$. Due to the design of this assay, drugs are diluted 20-fold between virus production and infection.

\section{Results}

DTG dose-response curve. We measured the DTG dose-response relationship using a single-round infectivity assay (see Methods), which allows for more accurate determination of pharmacodynamic parameters than do multiple-round assays (24). When plotted on a semi-log axis (Figure 1A, top), the DTG dose-response curve is sigmoidal. However, the same dose-response relationship is represented more informatively on a median-effect plot (Figure 1A, bottom), which presents efficacy on a logarithmic scale (10). The slope parameter is easily determined from the median-effect plot. The DTG dose-response curve has a slope of $1.3 \pm 0.1$, significantly higher $(P=0.0003)$ than the slope of 1 that is characteristic of raltegravir, a firstgeneration InSTI $(10,11)$. IIP, which equals the number of logs by which a single round of infection is reduced in the presence of drug(s), is given by the formula: $I I P=\log \left[1+\left(D / I C_{50}\right)^{m}\right]$, where $D$ is the drug concentration, and $m$ is the slope (10). For DTG at maximal clinical concentration $\left(\mathrm{C}_{\max }\right), \mathrm{IIP}=2.88$. The inhibition produced by DTG at $\mathrm{C}_{\text {max }}$ is almost 5-fold higher than that of a hypothetical drug with the same $\mathrm{IC}_{50}$ and the slope $m=1$, characteristic of first-generation InSTIs (Figure 1A, gray curves).

DTG does not act late in the HIV-1 life cycle. To determine whether the efficacy of DTG partially reflects activity during late steps of the HIV-1 life cycle (virus assembly, release, and maturation), as is the case for ALLINIs, we designed a modified infectivity assay (Figure 1B, Methods). Maximally activated $\mathrm{CD}^{+} \mathrm{T}$ lymphoblasts were infected with 1 of 2 different inocula containing the same concentrations of drug and virus. The only difference between the inocula was whether viral production occurred in the presence (inoculum A) or absence (inoculum B) of integrase inhibitors. We used raltegravir as a negative control for late life cycle activity and the ALLINI BI-D, which inhibits virion maturation (16, 25), as a positive control. 
A

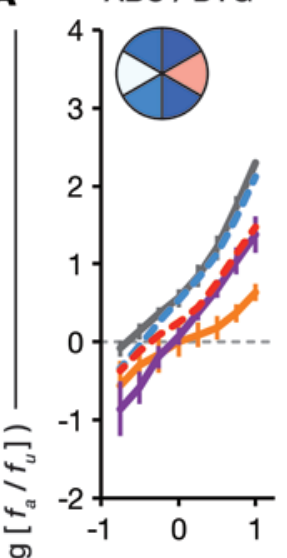

TDF / DTG

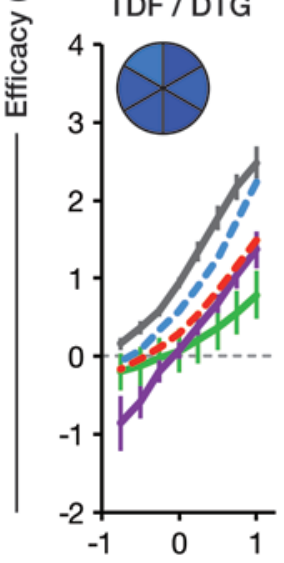

3TC / DTG

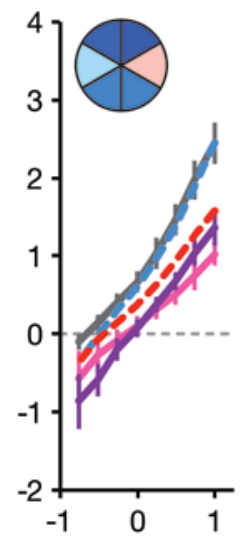

FTC / DTG

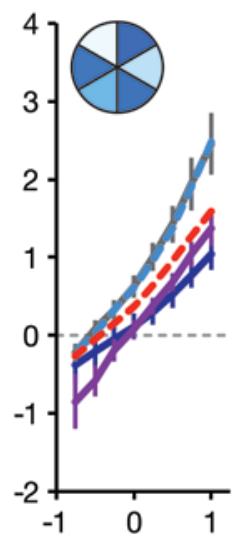

$\mathrm{ABC} / 3 \mathrm{TC}$

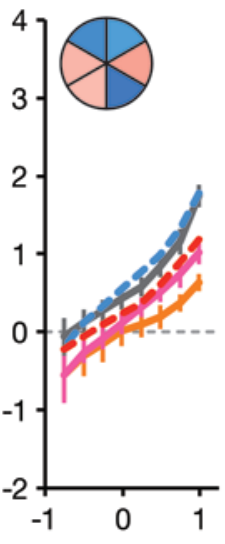

TDF / FTC

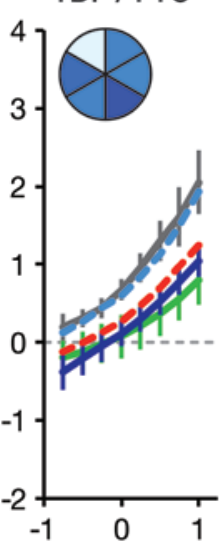

$\mathrm{ABC} / 3 \mathrm{TC} / \mathrm{DTG}$

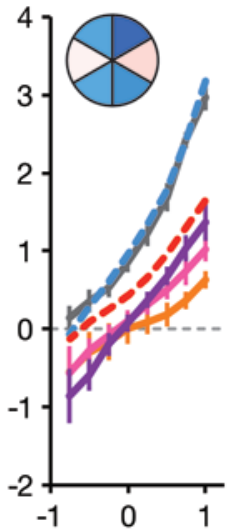

TDF / FTC / DTG

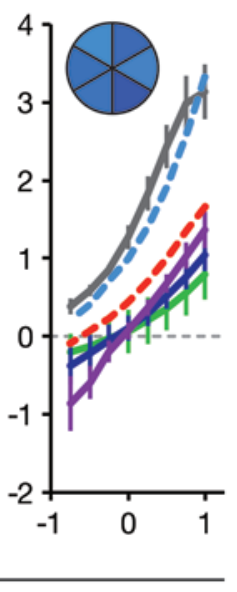

- $\mathrm{ABC}$

- 3ТС

- TDF

- FTC

- DTG

- combination

= - Bliss

- - Loewe
Degree of Independence

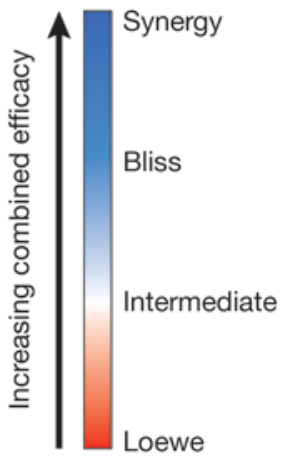

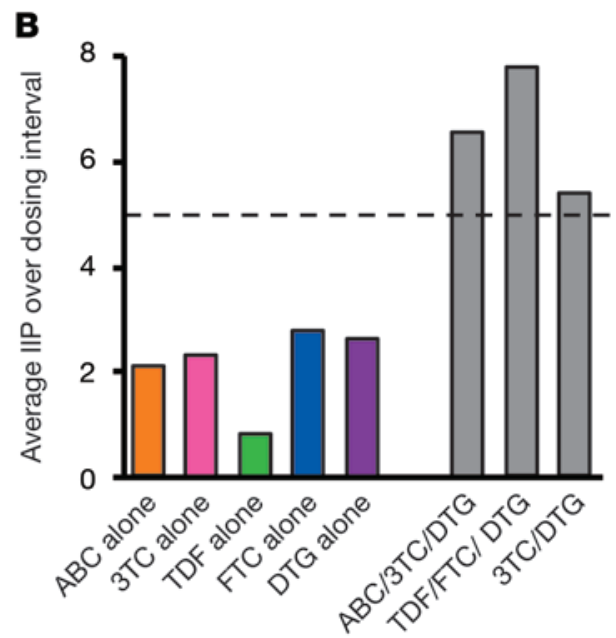

Figure 2. Efficacy of dolutegravir (DTG) alone and in combination with nucleoside analog reverse transcriptase inhibitors. (A) Median-effect curves for individual drugs and drug combinations. Solid lines show experimentally determined dose-response relationships, with color distinguishing the drug(s) used. The gray combination line shows the experimentally measured efficacy of the combination specified in the label above each chart. Dashed lines show the Loewe additivity (red) and Bliss independence (blue) model predictions for combined efficacy given the experimentally determined efficacies of individual drugs. Drug concentrations $[D]$ were chosen so that all drugs would have similar efficacy at $\left[D_{0}\right]:\left[D_{0, \text { DTC }}\right]=52.7 \mathrm{nM} ;\left[D_{0 . \mathrm{ABC}}\right]=558 \mathrm{nM} ;\left[D_{0,3 \mathrm{TC}}\right]=434$ $\mathrm{nM} ;\left[D_{0, T D F}\right]=354 \mathrm{nM} ;\left[D_{0, F T C}\right]=110 \mathrm{nM}$. Error bars show the standard deviation of 6 blood donors. Inset pie charts show degree of independence calculated for drug combinations, according to the color scale in the legend. Each pie slice shows the results from a different blood donor. (B) Average instantaneous inhibitory potential (IIP) over a 24-hour dosing period for individual drugs and their combinations. A dashed line at IIP = 5 shows the minimum IIP for a fully suppressive antiretroviral regimen (10). ABC, abacavir; 3TC, lamivudine; TDF, tenofovir disoproxil fumarate; FTC, emtricitabine.

The difference between virus produced in the presence versus the absence of BI-D is dramatic, with a greater than 2.5-log difference in efficacy at the highest concentration tested (Figure 1C). Inhibition of infection was readily detected when BI-D was present during virion maturation (inoculum A), but not when virus was combined with BI-D after viral production (inoculum B). This is consistent with reports that the $\mathrm{IC}_{50}$ of BI-D as a maturation inhibitor is 13 -fold lower than as an integrase inhibitor (16). In sharp contrast with the results obtained with BI-D, there was no difference in infectivity between virus produced in the presence or the absence of raltegravir, with less than 0.2-log difference between inocula A and B at all concentrations tested (Figure 1C, center). These contrasting results for raltegravir and BI-D demonstrate that this assay measures drug effects on late life cycle steps separately from effects on integration.

Using this system, we found no difference in infectivity between virus produced in the presence or the absence of DTG (Figure 1C). Antiretroviral drugs that act at multiple steps of the HIV-1 life cycle have nonlinear dose-response curves with steep slopes (12), which yield high efficacy at clinical concentrations (10-12). Both inocula A and B have DTG dose-response curves with slopes of $1.3 \pm 0.1$. Although this value is higher than the slope of 1 previously reported for first-generation InSTIs $(10,11)$, these experiments show that the difference does not reflect activity late in the viral life cycle. 
A

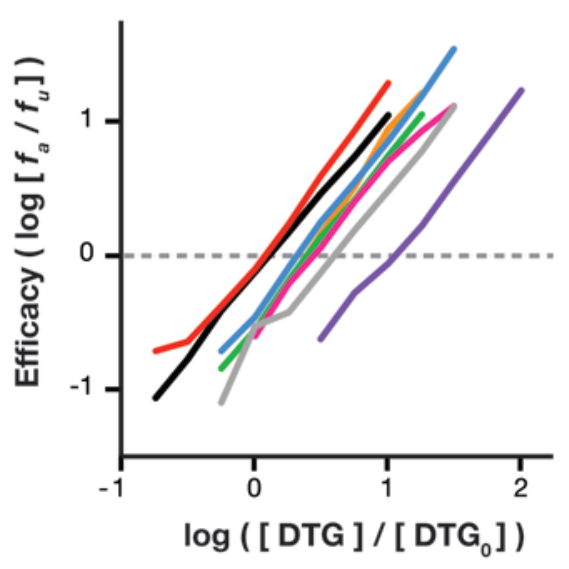

B
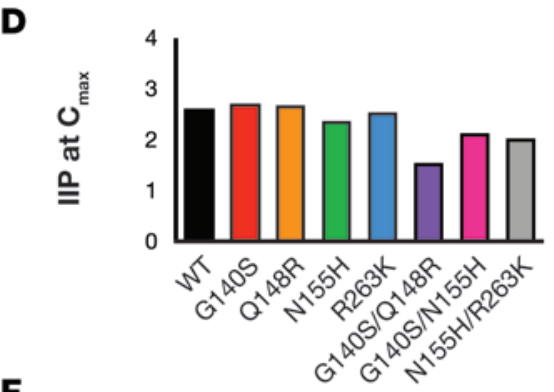

$\mathbf{F}$

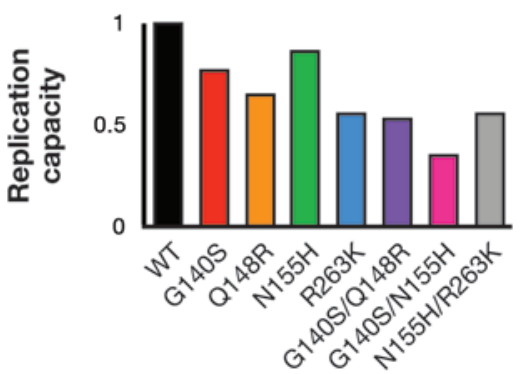

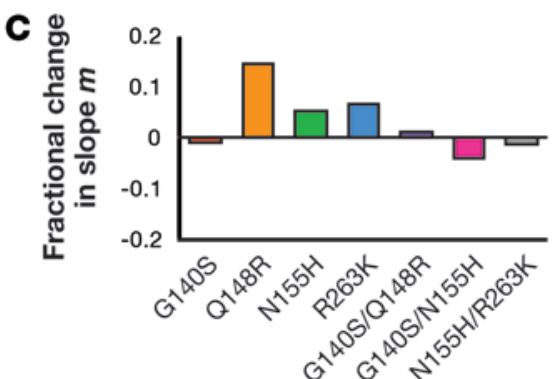

E

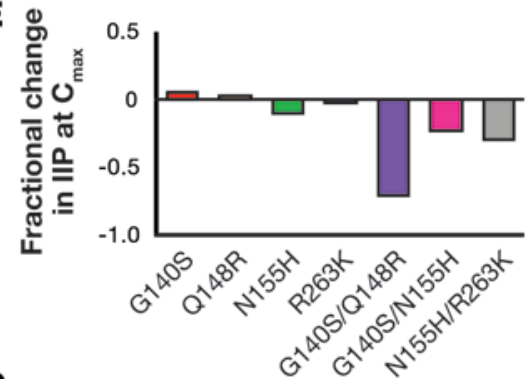

G

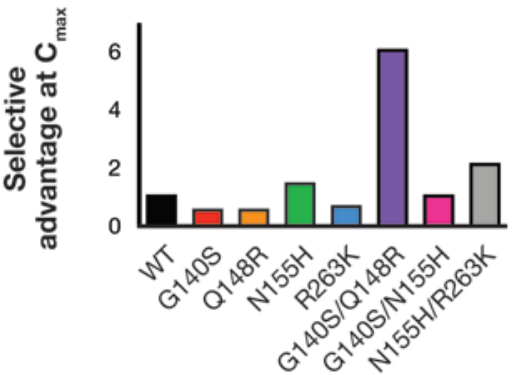

Figure 3. Pharmacodynamic properties of dolutegravir (DTC) against integrase strand transfer inhibitor resistance mutations. (A) Median-effect DTC dose-response curves for wild-type (WT) HIV- 1 and the indicated viral variants. The reference concentration $\left[D T G_{0}\right]=50 \mathrm{nM}$. The dotted line indicates $50 \%$ of maximal inhibition. (B) Fold change in IC ${ }_{50}$ of DTC dose-response curves for drug-resistant viruses, compared with WT. (C) Fractional change in slope of DTC dose-response curves for drug-resistant viruses, compared with WT. (D) Instantaneous inhibitory potential (IIP) of DTC at the maximal clinical concentration $\left(C_{\max }\right)$ against WT and drug-resistant viruses. (E) Fractional change in IIP of DTC at $C_{\max }$ against drug-resistant viruses, compared with WT. (F) Replication capacity of WT and drug-resistant viruses, defined as the ratio of maximal infection levels in vitro for drug-resistant and WT virus. (C) Selective advantage of each virus at $C_{\text {max }}$, defined as the replication capacity of the drug-resistant virus multiplied by the ratio of the $f_{u}$ values for the drug-resistant and WT viruses (26). Viruses with a selective advantage greater than 1 replicate better than WT in the presence of a drug, while viruses with a selective advantage less than 1 replicate worse than WT. All panels show the average of independent experiments using cells from 5 different blood donors.

DTG interacts favorably with NRTIs. To determine the combinatorial pharmacodynamics of DTG, we quantified its activity in combination with nucleoside analog reverse transcriptase inhibitors (NRTIs). Figure 2A shows the dose-response relationships for DTG, abacavir (ABC), lamivudine (3TC), tenofovir disoproxil fumarate (TDF), and emtricitabine (FTC) separately and in combination. The 3-drug combinations shown (ABC/3TC/DTG and TDF/FTC/DTG) are recommended initial regimens (26).

Plotted alongside the empirical dose-response curves in Figure 2A are the Loewe (red dashed) and Bliss (blue dashed) model predictions for the combined effects of each combination. DI, a scale defined by the Loewe and Bliss models, describes drug interactions quantitatively; higher DI values indicate more mechanistic independence and higher regimen efficacy (11). Some drug combinations (gray curves) exhibit an intermediate DI with efficacy between the Loewe and Bliss predictions; other combinations exhibit synergy, defined as a higher combined efficacy than that predicted by the Bliss model. No antagonistic interactions (defined as combined efficacy lower than the Loewe model prediction) have been observed between antiretroviral drugs (11). Pie charts in Figure 2A show DI values for DTG/NRTI combinations. Although DI varies by donor, we found that DTG/ABC/3TC interact according to the Bliss model, and DTG/TDF/FTC interact synergistically. Both combinations show higher antiviral activity than that predicted by the Loewe additivity model, which is most commonly used to predict combined drug effects (11).

DTG-based regimens have sufficient efficacy to suppress viremia. IIP quantifies the number of logs by which a drug or drug combination reduces a single round of HIV-1 infection (10). IIP is a function of 
drug concentration(s), dose-response curve slope, and $\mathrm{IC}_{50}$. The IIP for a combination also depends on the interactions between drugs, quantified by DI. IIP correlates closely with clinical success for most antiretroviral drug combinations, although it has been shown to undervalue first-generation InSTIs. Regimens with a combined average IIP greater than 5 throughout the dosing interval typically can control HIV-1 infection in vivo (11). Figure $2 \mathrm{~B}$ shows average IIP for individual drugs and the combinations $\mathrm{DTG} / \mathrm{ABC} / 3 \mathrm{TC}$ and DTG/TDF/FTC. Both 3-drug combinations have an average IIP between 6 and 8 , representing 6-8 logs of inhibition of a single round of infection and consistent with excellent reported clinical suppression of viremia. Interestingly, the 2-drug combination DTG/3TC, which has shown promise clinically (27), also has an average IIP greater than 5 .

DTG has a high genetic barrier to resistance. Figure 3A shows DTG dose-response curves for viruses containing 1 or more InSTI RMs. Individual mutations, including R263K, have a modest effect, increasing the $\mathrm{IC}_{50}$ less than 3-fold (Figure 3B). In contrast, single $\mathrm{RMs}$ can increase the $\mathrm{IC}_{50}$ of first-generation InSTIs by more than an order of magnitude (28). Although RMs can dramatically impact the slopes of doseresponse curves for some antiretroviral drugs, the dose-response curve slopes of first-generation InSTIs are consistent across wild-type and drug-resistant viruses (28). We show here that DTG recapitulates this phenotype, as the curves in Figure 3A all have similar slopes (Figure 3C).

IIP, which is a function of dose-response curve slope and $\mathrm{IC}_{50}$, can be used to quantify the impact of RMs on the efficacy of DTG (Figure 3, D and E). Individual RMs have minimal impact on the IIP of DTG at $\mathrm{C}_{\max }$, and the change in IIP due to $2 \mathrm{RMs}$ is comparable to the effect of single RMs on first-generation InSTIs (26).

The ability of drug-resistant HIV-1 to replicate in the presence of drug depends on the magnitude of resistance and the fitness cost of the RM. Figure 3F shows the impact of InSTI RMs on viral fitness, defined as the ratio of in vitro infection levels for wild-type and mutated viruses. Figure $3 \mathrm{G}$ shows the selective advantage of each virus, which reflects both the magnitude and the fitness cost of resistance. Importantly, at $\mathrm{C}_{\max }$, single mutations produce no selective advantage over wild-type. This result is consistent with the rarity of clinical DTG resistance (29). The variant with the greatest selective advantage over wild-type at $\mathrm{C}_{\max }$ is G140S/Q148R. Together, these $2 \mathrm{RMs}$ cause a 10-fold increase in $\mathrm{IC}_{50}$ with only a moderate fitness cost. The G140S mutation, which confers no resistance on its own, is a compensatory mutation for other InSTI RMs (30). Clinical failure of DTG, which is not observed in adherent patients with no prior exposure to integrase inhibitors, does correlate with prior failure of first-generation InSTIs (6).

\section{Discussion}

The overwhelming clinical success and increasing popularity of DTG suggest the importance of applying a quantitative framework describing antiretroviral therapy $(10,11,26,31)$ to this second-generation InSTI. DTG shares many pharmacodynamic features with first-generation InSTIs, including efficacy restricted to the early HIV-1 life cycle, favorable interactions with NRTIs, and a linear median-effect dose-response curve with a slope that remains consistent despite RMs. However, DTG has a higher dose-response curve slope than first-generation InSTIs, which leads to higher predicted clinical efficacy. The IIP of DTG at its maximal clinical concentration is 2.98 , more than $60 \%$ higher than that of raltegravir (1.86). Additionally, DTG has a remarkably high barrier to resistance; none of the single RMs we tested conferred substantial selective advantage over wild-type. Importantly, our model predicts sufficient efficacy at clinical concentrations for both $\mathrm{ABC} / 3 \mathrm{TC} / \mathrm{DTG}$ and TDF/FTC/DTG to completely suppress viremia, consistent with the success of these 2 regimens.

\section{Methods}

Single-round phenotypic infectivity assay. The phenotypic assay used to quantify DTG efficacy alone and in combination against wild-type and mutant HIV-1 has been previously described $(10,11,28,32)$. Briefly, maximally activated primary $\mathrm{CD} 4^{+} \mathrm{T}$ lymphoblasts from healthy blood donors were treated with varying concentrations of $\operatorname{drug}(\mathrm{s})$ and incubated overnight. The cells were then spinoculated with the single-round fluorescent reporter virus NL4-3 $\triangle$ Env-EGFP and incubated for 3 days, after which the proportion of infected cells was quantified by flow cytometry.

Replication cycle assay. 293T cells were transfected using Lipofectamine 2000 (Life Technologies) with a plasmid carrying the proviral clone NL4-3 with EGFP in place of the env gene and a separate plasmid carrying the IIIB env gene to produce single-round fluorescent reporter virus. A second flask of 293T cells 
was mock transfected. Six to 7 hours after transfection, the cells were trypsinized and transferred to 96-well plates in media comprised of 45\% RPMI, 5\% FBS, 50\% human serum (Gemini), and 12 mM HEPES. Integrase inhibitors were added to half of the wells to produce the following conditions: (a) transfected 293 T cells incubated with drug, (b) transfected $293 \mathrm{~T}$ cells incubated without drug, (c) mock-transfected 293 T cells incubated with drug, and (d) mock-transfected 293T cells incubated without drug (Figure 1B).

After a 48-hour incubation, supernatant was recovered, centrifuged briefly to remove cellular debris, and transferred to a fresh plate. The conditions described above produced supernatant containing the following: (a) virus matured in the presence of drug, (b) virus matured in the absence of drug, (c) drug without virus, and (d) neither virus nor drug. Supernatants 1 and 4 were combined at equal volumes to yield inoculum A, virus produced in the presence of drug. Supernatants 2 and 3 were combined at equal volumes to yield inoculum B, virus and drug that had been incubated separately. Both combinations were used to infect phytohemagglutinin-activated primary $\mathrm{CD}^{+} \mathrm{T}$ lymphoblasts by spinoculation at $1,200 \mathrm{~g}$ and $37^{\circ} \mathrm{C}$ for 2 hours. Infection was measured after 72 hours by EGFP fluorescence on a FACSCalibur (BD).

Materials. The BI-D used in this study was synthesized by James Fuchs (Ohio State University, Columbus, Ohio, USA) and provided by Alan Engelman (Harvard University, Cambridge, Massachusetts, USA).

Statistics. The slopes of raltegravir and DTG were compared with a 2-tailed Mann-Whitney $U$ test. Previously published values were used for the slope, $\mathrm{IC}_{50}, \mathrm{C}_{\max }$, and half-life parameters of $\mathrm{ABC}, 3 \mathrm{TC}$, TDF, FTC (11), as well as the $C_{\max }$ and half-life of DTG (33). The IIP for each drug combination according to the Loewe, Bliss, or empirical estimate of combined drug efficacy was calculated for every minute over a 24-hour dosing interval assuming rapid drug penetration. Average IIP over the dosing interval was reported as the average of these values. The average IIP calculation was performed using a python script, which is available as Supplemental material; supplemental material available online with this article; doi:10.1172/ jci.insight.90033DS1.

Study approval. This study was approved by the Johns Hopkins Institutional Review Board. All study participants provided informed, written consent.

\section{Author contributions}

SBL designed and conducted the experiments and analysis. SBL and RFS wrote the manuscript.

\section{Acknowledgments}

This work was supported by the National Institutes of Health (R01 grant AI081600), the Howard Hughes Medical Institute, the Martin Delaney CARE and DARE Collaboratories (NIH grants AI096113, 1U19AI096109), an ARCHE Collaborative Research Grant from the Foundation for AIDS Research (amFAR 108165-50-RGRL), the Johns Hopkins Center for AIDS Research (P30AI094189), and the Bill and Melinda Gates Foundation.

Address correspondence to: Robert F Siliciano, 733 North Broadway, MRB Room 879, Baltimore, Maryland 21205, USA. Phone: 410.955.2958; E-mail: rsiliciano@jhu.edu.

1. van Lunzen J, et al. Once daily dolutegravir (S/GSK1349572) in combination therapy in antiretroviral-naive adults with HIV: planned interim 48 week results from SPRING-1, a dose-ranging, randomised, phase 2b trial. Lancet Infect Dis. 2012;12(2):111-118.

2. Walmsley SL, et al. Dolutegravir plus abacavir-lamivudine for the treatment of HIV-1 infection. N Engl J Med. 2013;369(19):1807-1818.

3. Raffi F, et al. Once-daily dolutegravir versus twice-daily raltegravir in antiretroviral-naive adults with HIV-1 infection (SPRING-2 study): 96 week results from a randomised, double-blind, non-inferiority trial. Lancet Infect Dis. 2013;13(11):927-935.

4. Clotet B, et al. Once-daily dolutegravir versus darunavir plus ritonavir in antiretroviral-naive adults with HIV-1 infection (FLAMINGO): 48 week results from the randomised open-label phase 3b study. Lancet. 2014;383(9936):2222-2231.

5. Bollen P, Reiss P, Schapiro J, Burger D. Clinical pharmacokinetics and pharmacodynamics of dolutegravir used as a single tablet regimen for the treatment of HIV-1 infection. Expert Opin Drug Saf. 2015;14(9):1457-1472.

6. Malet I, et al. Combination of two pathways involved in raltegravir resistance confers dolutegravir resistance. $J$ Antimicrob Chemother. 2015;70(10):2870-2880.

7. Liang J, Mesplède T, Oliveira M, Anstett K, Wainberg MA. The combination of the R263K and T66I resistance substitutions in HIV-1 integrase is incompatible with high-level viral replication and the development of high-level drug resistance. $J$ Virol. 2015;89(22):11269-11274.

8. Pham HT, Mesplède T, Wainberg MA. Effect on HIV-1 viral replication capacity of DTG-resistance mutations in NRTI/ NNRTI resistant viruses. Retrovirology. 2016;13(1):31. 
9. Chou TC. Derivation and properties of Michaelis-Menten type and Hill type equations for reference ligands. J Theor Biol. 1976;59(2):253-276

10. Shen L, et al. Dose-response curve slope sets class-specific limits on inhibitory potential of anti-HIV drugs. Nat Med. 2008;14(7):762-766.

11. Jilek BL, et al. A quantitative basis for antiretroviral therapy for HIV-1 infection. Nat Med. 2012;18(3):446-451.

12. Rabi SA, et al. Multi-step inhibition explains HIV-1 protease inhibitor pharmacodynamics and resistance. J Clin Invest. 2013;123(9):3848-3860.

13. Desimmie BA, et al. LEDGINs inhibit late stage HIV-1 replication by modulating integrase multimerization in the virions. Retrovirology. 2013;10:57.

14. Le Rouzic E, et al. Dual inhibition of HIV-1 replication by integrase-LEDGF allosteric inhibitors is predominant at the postintegration stage. Retrovirology. 2013;10:144.

15. Balakrishnan M, et al. Non-catalytic site HIV-1 integrase inhibitors disrupt core maturation and induce a reverse transcription block in target cells. PLoS ONE. 2013;8(9):e74163.

16. Jurado KA, et al. Allosteric integrase inhibitor potency is determined through the inhibition of HIV-1 particle maturation. Proc Natl Acad Sci USA. 2013;110(21):8690-8695.

17. Gupta K, et al. Allosteric inhibition of human immunodeficiency virus integrase: late block during viral replication and abnormal multimerization involving specific protein domains. J Biol Chem. 2014;289(30):20477-20488.

18. Loewe S, Muischnek H. Effect of combinations: mathematical basis of problem. Arch Exp Pathol Pharmakol. 1926;114:313-326.

19. Bliss C. The toxicity of poisons applied jointly. Ann Appl Biol. 1939;26(3):585-615.

20. Rhee SY, Gonzales MJ, Kantor R, Betts BJ, Ravela J, Shafer RW. Human immunodeficiency virus reverse transcriptase and protease sequence database. Nucleic Acids Res. 2003;31(1):298-303.

21. Shafer RW. Rationale and uses of a public HIV drug-resistance database. J Infect Dis. 2006;194 Suppl 1:S51-S58.

22. Quashie PK, et al. Characterization of the R263K mutation in HIV-1 integrase that confers low-level resistance to the secondgeneration integrase strand transfer inhibitor dolutegravir. J Virol. 2012;86(5):2696-2705.

23. Wainberg M, Anstett K, Mesplede T, Quashie P, Han Y, Oliveira M. The R263K mutation in HIV integrase that is selected by dolutegravir may actually prevent clinically relevant resistance to this compound. J Int AIDS Soc. 2014;17(4 Suppl 3):19518.

24. Ferguson NM, Fraser C, Anderson RM. Viral dynamics and anti-viral pharmacodynamics: rethinking in vitro measures of drug potency. Trends Pharmacol Sci. 2001;22(2):97-100

25. Fontana J, et al. Distribution and redistribution of HIV-1 nucleocapsid protein in immature, mature, and integrase-inhibited virions: a role for integrase in maturation. J Virol. 2015;89(19):9765-9780.

26. Panel on Antiretroviral Guidelines for Adults and Adolescents. Guidelines for the use of antiretroviral agents in HIV-1-infected adults and adolescents. AidsInfo. http://aidsinfo.nih.gov/contentfiles/lvguidelines/AdultandAdolescentGL.pdf. Updated July 14, 2016. Accessed October 21, 2016.

27. Sued OG, et al. Comparable viral decay in dual and triple dolutegravir-based antiretroviral therapy. Abstract presented at: CROI Conference; February 22-25, 2016; Boston, Massachusetts. http://www.croiconference.org/sessions/comparable-viral-decaydual-and-triple-dolutegravir-based-antiretroviral-therapy. Accessed October 21, 2016.

28. Sampah ME, Shen L, Jilek BL, Siliciano RF. Dose-response curve slope is a missing dimension in the analysis of HIV-1 drug resistance. Proc Natl Acad Sci USA. 2011;108(18):7613-7618.

29. Wainberg MA, Mesplede T. Implications for the future of the HIV epidemic if drug resistance against dolutegravir cannot occur in first-line therapy. J Int AIDS Soc. 2015;18:20824.

30. Shadrina OA, Zatsepin TS, Agapkina YY, Isaguliants MG, Gottikh MB. Influence of drug resistance mutations on the activity of HIV-1 subtypes A and B integrases: a comparative study. Acta Naturae. 2015;7(1):78-86.

31. Laskey SB, Siliciano RF. A mechanistic theory to explain the efficacy of antiretroviral therapy. Nat Rev Microbiol. 2014;12(11):772-780

32. Zhang H, et al. Novel single-cell-level phenotypic assay for residual drug susceptibility and reduced replication capacity of drugresistant human immunodeficiency virus type 1. J Virol. 2004;78(4):1718-1729.

33. ViiV Healthcare Company. TIVICAY (Dolutegravir) prescribing information. https://www.gsksource.com/pharma/content/ dam/GlaxoSmithKline/US/en/Prescribing_Information/Tivicay/pdf/TIVICAY-PI-PIL.PDF. Accessed October 21, 2016. 